\title{
CLASSES OF NON-HERMITIAN OPERATORS WITH REAL EIGENVALUES*
}

\author{
NATÁLIA BEBIANO ${ }^{\dagger}$, JOÃO DA PROVIDÊNCIA ${ }^{\ddagger}$, AND JOÃO P. DA PROVIDÊNCIA§
}

\begin{abstract}
Classes of non-Hermitian operators that have only real eigenvalues are presented. Such operators appear in quantum mechanics and are expressed in terms of the generators of the Weyl-Heisenberg algebra. For each non-Hermitian operator $A$, a Hermitian involutive operator $\hat{J}$ such that $A$ is $\hat{J}$-Hermitian, that is, $\hat{J} A=A^{*} \hat{J}$, is found. Moreover, we construct a positive definite Hermitian $Q$ such that $A$ is $Q$-Hermitian, allowing for the standard probabilistic interpretation of quantum mechanics. Finally, it is shown that the considered matrices are similar to Hermitian matrices.
\end{abstract}

Key words. Infinite matrices, pseudo-Hermitian matrices, creation and annihilation operators, Krein spaces.

AMS subject classifications. 47B50, 47A63.

1. Motivation. In [4] Bhatia studies families of matrices that have only real eigenvalues, mentioning that the behaviour of the eigenvalues of those matrices has similarities to those of Hermitian matrices. Such matrices appear in the investigation of vectorial hyperbolical differential equations. The purpose of this note is to present classes of infinite matrices [5] that are non-Hermitian but have real eigenvalues. This investigation is motivated by certain operators that appear in quantum mechanics and our approach to determine their eigenvalues and eigenvectors is inspired by Dirac's method.

The interest in the study of non-Hermitian Hamiltonians in physics has been related, in the past, with the interpretation of some properties, such as transfer phenomena, typical of open systems. At present, it is also associated with new kinds of quantum theories characterized by non-Hermitian Hamiltonians with $\mathcal{P} \mathcal{T}$-symmetry (the product of parity and time reversal) and real spectra, the recent developments being motivated by field-theoretic models, such as the Lee model ([3] and the refer-

${ }^{*}$ Received by the editors on June 10, 2009. Accepted for publication on July 31, 2010. Handling Editors: Roger A. Horn and Fuzhen Zhang.

†Departamento de Matemática, Universidade de Coimbra, P-3001-454 Coimbra, Portugal (bebiano@mat.uc.pt).

${ }^{\ddagger}$ Departamento de Física, Universidade de Coimbra, P-3004-516 Coimbra, Portugal (providencia@teor.fis.uc.pt).

${ }^{\S}$ Depatamento de Física, Universidade da Beira Interior, P-6201-001 Covilhã, Portugal (joaoppc@ubi.pt). 
ences therein). The results obtained originated a consistent extension of the standard quantum mechanics. The notion of $\mathcal{P} \mathcal{T}$-symmetry can be placed in a general mathematical context known as pseudo-Hermiticity, a concept studied in the Krein space framework.

For the sake of completeness we introduce some terminology. As usual, we will refer to operators and matrices interchangeably. The states of a quantum system are described by vectors of an appropriate Hilbert space $\mathcal{H}$ of finite or infinite dimension, where the inner product of two vectors $u, v$ is denoted by $\langle u, v\rangle$. The state of the physical system at the instant $t$ is given by

$$
u(t)=\mathrm{e}^{-i t H} u(0),
$$

or equivalently by

$$
i \frac{\mathrm{d} u}{d t}=H u,
$$

where the linear operator $H: \mathcal{H} \rightarrow \mathcal{H}$ is called the Hamiltonian of the system assumed to be defined in a dense domain $\operatorname{Dom} H$. The energy of the state $u$ is given by the Rayleigh quotient $R(u)=\langle H u, u\rangle /\langle u, u\rangle, u \neq 0$. In non-relativistic quantum mechanics, the Hamiltonian operator is Hermitian, that is, $H=H^{*}$ where $\langle H x, y\rangle:=\left\langle x, H^{*} y\right\rangle$ for any $x, y \in \mathcal{H}$ in the domain of $H$. Throughout, we use the terms Hermitian and self-adjoint indistinctly. However, some relativistic extensions of quantum mechanics, such as the Klein-Gordon theory [7], lead to Hamiltonian operators $H$ that are non-Hermitian. In such theories an involutive selfadjoint operator $P$ (i.e., $P^{2}$ is the identity) arises that renders the Hamiltonian $P$-Hermitian (also said $P$-selfadjoint or pseudo-Hermitian), that is, $P H=H^{*} P$. This operator endows $\mathcal{H}$, assumed for simplicity to be finite dimensional, with a Krein space structure defined by an indefinite inner product $\langle u, v\rangle_{P}:=v^{*} P u$ (cf. e.g., [1, 2] for the theory of indefinite inner product spaces). In indefinite inner product spaces, there exist nonvanishing vectors such that $\langle u, u\rangle_{P}=0$ (isotropic vectors) and the selfadjoint operators between these spaces may have nonreal eigenvalues. This mathematical structure is the natural context to formulate nonrelativistic quantum mechanics. A Hermitian involutive operator $P$ with signature $(r, n-r)$ (i.e., with $r$ positive eigenvalues and $n-r$ negative eigenvalues) allows for the definition of an indefinite norm $\|u\|_{P}^{2}:=\langle u, u\rangle_{P}=u_{1}^{2}+\cdots+u_{r}^{2}-u_{r+1}^{2}+\cdots-u_{n}^{2}$. However, this norm is not suitable for the standard probabilistic quantum mechanical interpretation. So the problem arises of finding a positive definite Hermitian operator $Q$ that can play the role of $P$ and moreover is compatible with the conventional interpretation of quantum mechanics.

The main aim of this paper is the investigation of two classes of non-Hermitian infinite matrices that have only real eigenvalues and complete systems of eigenvectors (so they are diagonalizable under a similarity transformation). For each matrix, we 
construct a Hermitian involutive operator $\hat{J}$ that renders it $\hat{J}$-Hermitian. It is also proved that this allows for the unambiguous definition of a positive definite norm operator suitable for the conventional quantum mechanical interpretation. These results are obtained in Section 3. In Section 2 some notation is fixed and auxiliary results are given. Finally, in Section 4 open questions are formulated.

2. Prerequisites. As usual, $L^{2}(\mathbb{R})$ denotes the Hilbert space of square-integrable complex valued functions of a real variable $x$ endowed with the usual inner product

$$
\langle\psi, \phi\rangle=\int_{-\infty}^{+\infty} \bar{\phi}(x) \psi(x) \mathrm{d} x .
$$

We denote by $l^{2}$ the Hilbert space of complex valued sequences $\left\{x_{n}\right\}_{0}^{+\infty}$, such that the series $\sum_{n=0}^{+\infty}\left|x_{n}\right|^{2}$ converges, endowed with the usual inner product $\langle x, y\rangle=$ $\sum_{k=0}^{+\infty} \bar{y}_{k} x_{k}$. Throughout the paper, operation that are well-defined on $l^{2}$ for bounded operators (such as commutation) are used in the context of unbounded operators [5], a short explanation of their applicability being given.

For the sake of completeness, we briefly describe Dirac's approach to determine the eigenvalues and eigenvectors of the harmonic oscillator Hamiltonian $H: L^{2}(\mathbb{R}) \rightarrow$ $L^{2}(\mathbb{R})$ defined on a dense domain as follows,

$$
H:=\frac{1}{2}\left(\hat{p}^{2}+\hat{x}^{2}\right) .
$$

In physics, $\hat{p}: L^{2}(\mathbb{R}) \rightarrow L^{2}(\mathbb{R})$ is the differential operator $f(x) \rightarrow-i(\mathrm{~d} f / \mathrm{d} x)$ with dense domain Dom $\hat{p}$ and $\hat{x}: L^{2}(\mathbb{R}) \rightarrow L^{2}(\mathbb{R})$ is the multiplicative operator $f(x) \rightarrow$ $x f(x)$, with dense domain Dom $\hat{x}$. For an arbitrary $f \in \operatorname{Dom} \hat{p} \cap \operatorname{Dom} \hat{x} \subset L^{2}(\mathbb{R})$, let the commutator $[\hat{p}, \hat{x}]$ be defined as follows

$$
[\hat{p}, \hat{x}] f:=(\hat{p} \hat{x}-\hat{x} \hat{p}) f .
$$

It can be easily seen that the above operators satisfy the quantum condition

$$
[\hat{p}, \hat{x}]=-i \hat{1}
$$

where $\hat{1}: L^{2}(\mathbb{R}) \rightarrow L^{2}(\mathbb{R})$ denotes the identity operator. The following relations also hold

$$
[\hat{p},[\hat{p}, \hat{x}]]=[\hat{x},[\hat{p}, \hat{x}]]=0 .
$$

The Weyl-Heisenberg algebra is the algebra generated by $\hat{p}, \hat{x}, \hat{1}$. This algebra is equivalently generated by the creation and annihilation operators $\hat{a}^{*}$ and $\hat{a}$, respectively, defined by the linear combinations

$$
\hat{a}:=\frac{1}{\sqrt{2}}(\hat{x}+i \hat{p}), \quad \hat{a}^{*}:=\frac{1}{\sqrt{2}}(\hat{x}-i \hat{p}) .
$$


As the notation conveys, $\hat{a}^{*}$ is the adjoint operator of $\hat{a}$ and these operators satisfy the commutation relations

$$
\left[\hat{a}, \hat{a}^{*}\right]=\hat{1}, \quad[\hat{a}, \hat{1}]=0, \quad\left[\hat{a}^{*}, \hat{1}\right]=0,
$$

being the commutation defined as previously. The harmonic oscillator Hamiltonian is Hermitian and so its eigenvalues are real. Following Dirac's approach, we determine them with the help of the Weyl-Heisenberg algebra as shown above. One easily finds that $H$ may be written in terms of the creation and annihilation operators as follows:

$$
H=\hat{a}^{*} \hat{a}+\frac{1}{2} \hat{1} .
$$

We determine the vector $\phi_{0}$ such that $\hat{a} \phi_{0}=0$ by solving the differential equation

$$
\left(x+\frac{\mathrm{d}}{\mathrm{d} x}\right) \phi_{0}(x)=0 .
$$

We easily obtain $\phi_{0}=K_{0} \mathrm{e}^{-\frac{1}{2} x^{2}}$, with $K_{0}$ a real constant, and so $H \phi_{0}=\frac{1}{2} \phi_{0}$. For $K_{0}=\pi^{-\frac{1}{4}}$, we get $\left\langle\phi_{0}, \phi_{0}\right\rangle=1$. It can be easily verified that if $H \psi=\lambda \psi, \psi \neq 0$, then

$$
\begin{gathered}
H \hat{a} \psi=(\lambda-1) \hat{a} \psi, \quad \hat{a} \psi \neq 0, \\
H \hat{a}^{*} \psi=(\lambda+1) \hat{a}^{*} \psi, \psi \neq 0 .
\end{gathered}
$$

On the other hand, the functions

$$
\phi_{n}(x)=K_{n}\left(x-\frac{\mathrm{d}}{\mathrm{d} x}\right)^{n} \mathrm{e}^{-\frac{1}{2} x^{2}}
$$

where $K_{n}=\pi^{-\frac{1}{4}}\left(n ! 2^{n}\right)^{-\frac{1}{2}}$ is a real normalization constant, are eigenvectors associated with distinct eigenvalues. Indeed,

$$
H \phi_{n}=\left(n+\frac{1}{2}\right) \phi_{n}, \quad n=0,1,2, \ldots
$$

For $K_{n}=\pi^{-\frac{1}{4}}\left(n ! 2^{n}\right)^{-\frac{1}{2}}$, the eigenvectors $\phi_{n}$ are orthonormal, that is, $\left\langle\phi_{n}, \phi_{m}\right\rangle=\delta_{n m}$, $n, m=0,1,2, \ldots\left(\delta_{i j}\right.$ denotes the Kronecker symbol $)$. It is a well known fact that the eigenvectors form a complete system [6].

\section{Non-Hermitian operators with real eigenvalues.}


3.1. The extended harmonic oscillator. Let $H_{\beta}: L^{2}(\mathbb{R}) \rightarrow L^{2}(\mathbb{R})$ be an operator with a dense domain $\operatorname{Dom} H_{\beta}$, defined by

$$
H_{\beta}:=\frac{\beta}{2}\left(\hat{p}^{2}+\hat{x}^{2}\right)+i \sqrt{2} \hat{p}=\beta \hat{a}^{*} \hat{a}+\left(\hat{a}-\hat{a}^{*}\right)+\frac{\beta}{2} \hat{1}, \quad \beta>0 .
$$

This operator is the so called extended harmonic oscillator. Although $H_{\beta}$ is nonHermitian, it is nevertheless $P$-Hermitian, i.e., $P H_{\beta}=H_{\beta}^{*} P$, where $P: L^{2}(\mathbb{R}) \rightarrow$ $L^{2}(\mathbb{R})$ is the parity operator defined by $\operatorname{Pf}(x)=f(-x)$. This operator performs spatial reflections, so that it has the effect $p \rightarrow-p$ and $x \rightarrow-x$, and is Hermitian and involutive.

Proposition 3.1. The matrix representation of the annihilation operator $\hat{a}$ in (2.1) in the basis of the harmonic oscillator (2.3) is the infinite matrix

$$
\breve{a}=\left[\begin{array}{ccccc}
0 & \sqrt{1} & 0 & 0 & \cdots \\
0 & 0 & \sqrt{2} & 0 & \cdots \\
0 & 0 & 0 & \sqrt{3} & \cdots \\
0 & 0 & 0 & 0 & \cdots \\
\vdots & \vdots & \vdots & \vdots & \ddots
\end{array}\right] .
$$

Proof. By straightforward computations, we may conclude that

$$
\left[\hat{a}, \hat{a}^{*}\right]=\hat{1}, \quad\left[\hat{a}, \hat{a}^{* n}\right]=\left[\hat{a}, \hat{a}^{*}\right] \hat{a}^{* n-1}+\hat{a}^{*}\left[\hat{a}, \hat{a}^{*}\right] \hat{a}^{* n-2}+\cdots+\hat{a}^{* n-1}\left[\hat{a}, \hat{a}^{*}\right]=n \hat{a}^{* n-1} .
$$

Having in mind (2.3) we find

$$
\begin{aligned}
& \hat{a} \phi_{0}=0 \\
& \hat{a} \phi_{n}=K_{n} \hat{a} \hat{a}^{* n} \mathrm{e}^{-\frac{1}{2} x^{2}}=K_{n}\left[\hat{a}, \hat{a}^{* n}\right] \mathrm{e}^{-\frac{1}{2} x^{2}}=n K_{n} \hat{a}^{*(n-1)} \mathrm{e}^{-\frac{1}{2} x^{2}}=\sqrt{n} \phi_{n-1}, \quad n \geq 1,
\end{aligned}
$$

and the result clearly follows.

Proposition 3.2. The matrix representation of the creation operator $\hat{a}^{*}$ in (2.1) in the basis of the harmonic oscillator (2.3) is the infinite matrix $\breve{a}^{*}$.

Proof. We have

$$
\hat{a}^{*} \phi_{n}=K_{n} \hat{a}^{*} \hat{a}^{* n} \mathrm{e}^{-\frac{1}{2} x^{2}}=n K_{n} \hat{a}^{*(n+1)} \mathrm{e}^{-\frac{1}{2} x^{2}}=\sqrt{n+1} \phi_{n+1} .
$$

We find that, in the basis $(2.3), H_{\beta}$ is represented by the matrix

$$
M_{\beta}=\beta \breve{a}^{*} \breve{a}+\left(\breve{a}-\breve{a}^{*}\right)+\frac{\beta}{2} I,
$$

where $I$ denotes the infinite diagonal matrix $I=\operatorname{diag}(1,1,1, \cdots)$, that is, 


$$
M_{\beta}=\left[\begin{array}{ccccc}
\frac{1}{2} \beta & \sqrt{1} & 0 & 0 & \ldots \\
-\sqrt{1} & \frac{3}{2} \beta & \sqrt{2} & 0 & \ldots \\
0 & -\sqrt{2} & \frac{5}{2} \beta & \sqrt{3} & \ldots \\
0 & 0 & -\sqrt{3} & \frac{7}{2} \beta & \ldots \\
\vdots & \vdots & \vdots & \vdots & \ddots
\end{array}\right]
$$

The collection of matrices

$$
\mathcal{S}=\left\{\alpha\left(\breve{a}-\breve{a}^{*}\right)+\beta\left(\breve{a}^{*} \breve{a}+I / 2\right), \alpha \in \mathbb{R}, \beta>0\right\}
$$

with the infinite zero matrix form a convex cone of the real vector space generated by $\breve{a}-\breve{a}^{*}$ and $\breve{a}^{*} \breve{a}+I / 2$. Obviously, an analogous situation occurs if $\beta<0$. We show that the matrices of $\mathcal{S}$ have real eigenvalues, complete systems of eigenvectors and moreover they are $Q$-Hermitian, for $Q$ a positive definite Hermitian operator. This result is proved in the next theorem, where we consider a matrix of $\mathcal{S}$ with $\alpha=1$ and $\beta=2$ for simplicity.

Theorem 3.3. Consider the infinite matrix $M=2 \breve{a} \breve{a}^{*}+\left(\breve{a}-\breve{a}^{*}\right)+I$. Then

(i) The point spectrum of the matrix $M$ is the set

$$
\sigma(M)=\left\{\frac{3}{2}+2 j: j=0,1,2, \ldots\right\}
$$

and the vector

$$
v_{j}=K_{j}\left(\breve{a}^{*}+\frac{I}{2}\right)^{j}\left(1, \frac{1}{2 \sqrt{1}}, \frac{1}{2^{2} \sqrt{1 \cdot 2}}, \frac{1}{2^{3} \sqrt{1 \cdot 2 \cdot 3}}, \cdots\right)^{T} \in l^{2},
$$

$K_{j}$ being a normalization constant, is a right eigenvector associated with the eigenvalue $\frac{3}{2}+2 j$, for $j=0,1,2, \ldots$ This eigensystem is complete.

(ii) We have $M=\mathrm{e}^{S}\left(2 a^{*} \breve{a}+\frac{3}{2} I\right) \mathrm{e}^{-S}$, where $S=\frac{1}{2}\left(\breve{a}+\breve{a}^{*}\right)$. Moreover, $M$ is $Q$-Hermitian for $Q$ the positive Hermitian operator $Q=\mathrm{e}^{-\left(\breve{a}+\breve{a}^{*}\right)}$.

Proof. Consider the matrix $M=2 \breve{a}^{*} \breve{a}+\breve{a}-\breve{a}^{*}+I$. Let

$$
\breve{c}^{\ddagger}=\breve{a}^{*}+\frac{1}{2} I, \quad \breve{c}=\breve{a}-\frac{1}{2} I .
$$

Then we obtain

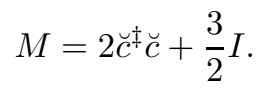


Let $\left[\breve{c}, \breve{c}^{\ddagger}\right]:=\breve{c} \breve{c^{\ddagger}}-\breve{c}^{\ddagger} \breve{c}$. It may be easily seen that $\left[\breve{c}, \breve{c}^{\ddagger}\right] f=f$ for any $f \in L_{2}(\mathbb{R})$ and so the following holds

$$
\left[\breve{c}, \breve{c}^{\ddagger}\right]=I
$$

Similarly, it may be shown that

$$
\left[M, \breve{c} \breve{c}^{\ddagger}\right]=2 \breve{c}^{\ddagger}, \quad[M, \breve{c}]=-2 \breve{c} .
$$

If $v \in l^{2}$ and $\lambda \in \mathbb{R}$ are such that $M v=\lambda v$, then by (3.6) we get

$$
M \breve{c}^{\ddagger} v=\left[M, \breve{c}^{\ddagger}\right] v+\breve{c}^{\ddagger} M v=(\lambda+2) \breve{c}^{\ddagger} v, \quad M \breve{c} v=(\lambda-2) \breve{c} v .
$$

If $\breve{c} v \neq 0$, then $\breve{c} v$ is an eigenvalue of $M$ associated to the eigenvalue $\lambda-2$. We observe that $\breve{c}^{\ddagger} v$ is different from 0 and so is an eigenvector of $M$ associated to the eigenvalue $\lambda+2$.

We claim that there exists a nonnegative integer $j$ such that $\breve{c}^{j} v=0$. We will accept this for the moment and the claim will be proved in (ii). It may be easily checked that, for $v_{0}=\left(1, \frac{1}{2 \sqrt{1}}, \frac{1}{2^{2} \sqrt{1 \cdot 2}}, \frac{1}{2^{3} \sqrt{1 \cdot 2 \cdot 3}}, \cdots\right)^{T} \in l^{2}$, we have $\breve{c} v_{0}=0$. Thus,

$$
M\left(\breve{c}^{\ddagger}\right)^{j} v_{0}=\left(2 j+\frac{3}{2}\right)\left(\breve{c}^{\ddagger}\right)^{j} v_{0}, \quad j=0,1,2,3, \ldots,
$$

and so $v_{j}=\left(\breve{c}^{\ddagger}\right)^{j} v_{0}$. Taking into account (3.4), we obtain the asserted expression for $v_{j}$ in (i).

The eigenvectors $v_{j}, j=0,1,2,3, \ldots$, of $M$ have been expressed in terms of the basis elements $e_{j}, j=0,1,2,3, \ldots$, with $e_{0}=(1,0,0, \ldots)^{T}, e_{1}=(0,1,0,0, \ldots)^{T}$, $e_{2}=(0,0,1,0, \ldots)^{T}, \ldots$, being

$$
\breve{a} e_{0}=0, e_{1}=\breve{a}^{*} \sqrt{1 !} e_{0}, e_{2}=\breve{a}^{* 2} \sqrt{2 !} e_{0}, \ldots
$$

By inverting the transformation (3.4), we obtain

$$
\breve{a}^{*}=\breve{c}^{\ddagger}-\frac{1}{2} I, \quad \breve{a}=\breve{c}+\frac{1}{2} I .
$$

Thus, we are able to express the vectors $e_{j}$ in terms of the eigenvectors $v_{j}$. This proves that these eigenvectors form a complete basis and (i) follows.

Now we prove (ii). Let $S=\frac{1}{2}\left(\breve{a}+\breve{a}^{*}\right)$, where $\breve{a}$ is given in (3.1). By definition $\mathrm{e}^{S}=I+S+\frac{S^{2}}{2 !}+\cdots$ and $\mathrm{e}^{S} \breve{a} \mathrm{e}^{-S}$ is given by the formal series expansion

$$
\mathrm{e}^{S} \breve{a} \mathrm{e}^{-S}=\breve{a}+[S, \breve{a}]+\frac{1}{2}[S,[S, \breve{a}]]+\cdots
$$


This series has only a finite number of nonvanishing terms, because $\left[\breve{a}, \breve{a}^{*}\right]=\hat{1}$. We notice that since the series expansion have a finite number of nonvanishing terms, the domain of the unbounded operator in the left side is equal to the domain of the operator in the right side, namely the subspace of $l^{2}$ constituted by the vectors $\left(x_{1}, x_{2}, \ldots\right)^{T}$ such that the series $\sum_{j=1}^{\infty} j\left|x_{j}\right|^{2}$ converges.

Having (2.2) in mind, we find

$$
\mathrm{e}^{S} \breve{a} \mathrm{e}^{-S}=\breve{a}-\frac{1}{2} I
$$

Analogously,

$$
\mathrm{e}^{S} \breve{a}^{*} \mathrm{e}^{-S}=\breve{a}^{*}+\frac{1}{2} I
$$

Thus,

$$
\mathrm{e}^{S}\left(2 \breve{a}^{*} \breve{a}+\frac{3}{2} I\right) \mathrm{e}^{-S}=2 \breve{a}^{*} \breve{a}+\breve{a}-\breve{a}^{*}+I=M
$$

We have shown that for $M_{0}=2 \breve{a}^{*} \breve{a}+\frac{3}{2} I$, and obtain $\mathrm{e}^{S} M_{0} \mathrm{e}^{-S}=M$. We clearly have $\mathrm{e}^{-S} M_{0} \mathrm{e}^{S}=M^{*}$, or, equivalently,

$$
M_{0}=\mathrm{e}^{-S} M \mathrm{e}^{S}=\mathrm{e}^{S} M^{*} \mathrm{e}^{-S} .
$$

Hence

$$
\mathrm{e}^{-2 S} M=M^{*} \mathrm{e}^{-2 S}
$$

Finally, we prove the claim. In fact, since $M$ is similar to the harmonic oscillator Hamiltonian $M_{0}=2 \breve{a}^{*} \breve{a}+\frac{3}{2} I, M$ and $M_{0}$ have the same eigenvalues. The point spectrum of $M_{0}$ is bounded from below (cf. [7]), so the claim follows.

REMARK 3.4. A left eigenvector of $M$ satisfies

$$
w_{j}^{*} M=\left(2 j+\frac{3}{2}\right) w_{j}^{*}, \quad j=0,1,2, \ldots, \quad w_{0}^{*} \breve{c}^{\ddagger}=0, \quad w_{j}^{*} \breve{c}=\kappa_{j} w_{j+1}^{*},
$$

for some $\kappa_{j}>0$, and is such that

$$
w_{j}=K_{j}\left(\breve{a}^{*}-\frac{I}{2}\right)^{j}\left(1,-\frac{1}{2 \sqrt{1}}, \frac{1}{2^{2} \sqrt{1 \cdot 2}},-\frac{1}{2^{3} \sqrt{1 \cdot 2 \cdot 3}}, \cdots\right)^{T} \in l^{2} .
$$

Moreover, for $K_{j}=\sqrt{\frac{\mathrm{e}}{j !}}$, we have $w_{j}^{*} v_{l}=\delta_{k l}$, i.e., the left and right eigenvectors are orthonormal. 
3.2. The Swanson-Jones Hamiltonian. Next, we consider an operator of a type investigated by Swanson [9] and Jones [8], namely the non-Hermitian Hamiltonian $H_{\theta}: L^{2}(\mathbb{R}) \rightarrow L^{2}(\mathbb{R})$

$$
H_{\theta}:=\frac{1}{2}\left(\hat{p}^{2}+\hat{x}^{2}\right)-\frac{i}{2} \tan 2 \theta\left(\hat{p}^{2}-\hat{x}^{2}\right),
$$

where $\theta$ is a real constant, $-\frac{\pi}{4}<\theta<\frac{\pi}{4}$, and whose domain is assumed to be dense. In terms of creation and annihilation operators $\hat{a}^{*}, \hat{a}$ (cf. eq. (2.1)), we easily find

$$
H_{\theta}=\hat{a}^{*} \hat{a}+i \frac{\tan 2 \theta}{2}\left(\hat{a}^{* 2}+\hat{a}^{2}\right)+\frac{1}{2} \hat{1} .
$$

In the basis of the mentioned eigenvectors of the harmonic oscillator, this operator has the following matrix representation:

$$
M_{\theta}=\left[\begin{array}{cccccc}
\frac{1}{2} & 0 & i \kappa \sqrt{1 \cdot 2} & 0 & 0 & \ldots \\
0 & \frac{3}{2} & 0 & i \kappa \sqrt{2 \cdot 3} & 0 & \ldots \\
i \kappa \sqrt{1 \cdot 2} & 0 & \frac{5}{2} & 0 & i \kappa \sqrt{3 \cdot 4} & \ldots \\
0 & i \kappa \sqrt{2 \cdot 3} & 0 & \frac{7}{2} & 0 & \ldots \\
0 & 0 & i \kappa \sqrt{3 \cdot 4} & 0 & \frac{9}{2} & \ldots \\
\vdots & \vdots & \vdots & \vdots & \vdots & \ddots
\end{array}\right],
$$

with $\kappa=\frac{1}{2} \tan 2 \theta$. Consider the collection of matrices

$$
\mathcal{R}=\left\{i \alpha\left(\hat{a}^{* 2}+\hat{a}^{2}\right)+\beta\left(\hat{a}^{*} \hat{a}+I / 2\right), \alpha \in \mathbb{R}, \beta>0\right\} .
$$

The union of the set $\mathcal{R}$ with the infinite zero matrix forms a convex cone of the real vector space generated by $\hat{a}^{*} \hat{a}+I / 2$ and $\hat{a}^{* 2}+\hat{a}^{2}$. Obviously, an analogous situation occurs if $\beta<0$. We show that the matrices of $\mathcal{R}$ have similar properties to those of $\mathcal{S}$. Indeed, they have real eigenvalues, complete systems of eigenvectors and they are $Q$-Hermitian, for $Q$ a positive Hermitian operator. This result is proved in the next theorem, where we consider a matrix in $\mathcal{R}$ with $\alpha=2$ and $\beta=2$ for simplicity.

TheOREM 3.5. Consider the infinite matrix $M=2 M_{\frac{\pi}{8}}$, where $M_{\frac{\pi}{8}}$ is of the form (3.7). Then

(i) The point spectrum of $M$ is $\sigma(M)=\{\sqrt{2}(1+2 j): j=0,1,2, \ldots\}$, and the $l^{2}$-vector $v_{j}=K_{j}\left(\breve{a}^{*}+i \gamma \breve{a}\right)^{j}\left(1,0,-i \gamma \sqrt{\frac{1}{2}}, 0,(-i \gamma)^{2} \sqrt{\frac{1 \cdot 3}{2 \cdot 4}}, 0,(-i \gamma)^{3} \sqrt{\frac{1 \cdot 3 \cdot 5}{2 \cdot 4 \cdot 6}} \cdots\right)^{T}$ with $\gamma=\tan \frac{\pi}{8}$ and $K_{j}$ a normalization constant, is a right eigenvector associated with the eigenvalue $\sqrt{2}(2 j+1)$, for $j=0,1,2, \ldots$ This eigensystem is complete. 
(ii) We have $M=\mathrm{e}^{S} \sqrt{2}\left(2 \breve{a}^{*} \breve{a}+I\right) \mathrm{e}^{-S}$, where $S=\frac{\pi}{16} i\left(\breve{a}^{2}-\breve{a}^{* 2}\right)$. Moreover, $M$ is $Q$-Hermitian for $Q$ the positive Hermitian operator $Q=\mathrm{e}^{-\frac{\pi}{8} i\left(\breve{a}^{2}-\breve{a}^{* 2}\right)}$.

Proof. Consider the matrix $M=2 \breve{a}^{*} \breve{a}+i\left(\breve{a}^{2}+\breve{a}^{* 2}\right)+I$. Let

$$
\breve{c}^{\ddagger}=\cos \frac{\pi}{8} \breve{a}^{*}+i \sin \frac{\pi}{8} \breve{a}, \quad \breve{c}=\cos \frac{\pi}{8} \breve{a}+i \sin \frac{\pi}{8} \breve{a}^{*},
$$

Thus,

$$
M=\sqrt{2}\left(2 \breve{c}^{\ddagger} \breve{c}+I\right),
$$

and the following commutation relations are valid

$$
\left[\breve{c}, \breve{c}^{\ddagger}\right]=I, \quad\left[M, \breve{c}^{\ddagger}\right]=2 \sqrt{2} \breve{c}^{\ddagger}, \quad[M, \breve{c}]=-2 \sqrt{2} \breve{c} .
$$

If $v \in l^{2}, \lambda \in \mathbb{R}$ are such that $M v=\lambda v$, then

$$
M \breve{c}^{\ddagger} v=\left[M, \breve{c}^{\ddagger}\right] v+\breve{c}^{\ddagger} M v=(\lambda+2 \sqrt{2}) \breve{c}^{\ddagger} v, \quad M \breve{c} v=(\lambda-2 \sqrt{2}) \breve{c} v .
$$

As in the previous theorem, we can show that there exists a nonnegative integer $j$ such that $\breve{c}^{j} v=0$ It may be easily checked that, for $v_{0}=\left(1,0,-i \gamma \sqrt{\frac{1}{2}}, 0,(-i \gamma)^{2} \sqrt{\frac{1 \cdot 3}{2 \cdot 4}}, 0\right.$, $\left.(-i \gamma)^{3} \sqrt{\frac{1 \cdot 3 \cdot 5}{2 \cdot 4 \cdot 6}}, \cdots\right)^{T} \in l^{2}, \quad \gamma=\tan \frac{\pi}{8}$, we have $\breve{c} v_{0}=0$. Thus,

$$
M\left(\breve{c}^{\ddagger}\right)^{j} v_{0}=\sqrt{2}(2 j+1)\left(\breve{c}^{\ddagger}\right)^{j} v_{0}, \quad j=0,1,2,3, \ldots,
$$

and this yields the expression for $v_{j}$ in (i). Using analogous arguments to those in the proof of Theorem 3.1, it can be shown that this eigensystem is complete.

Now we prove (ii). Let $S=\frac{\pi}{16} i\left(\breve{a}^{2}-\breve{a}^{* 2}\right)$. Similarly to the proof of Theorem 3.1 (ii), we easily find

$$
[S, \breve{a}]=\frac{\pi}{8} i \breve{a}^{*}, \quad\left[S, \breve{a}^{*}\right]=\frac{\pi}{8} i \breve{a} .
$$

Thus,

$$
\mathrm{e}^{S} \breve{a} \mathrm{e}^{-S}=\cos \frac{\pi}{8} \breve{a}+i \sin \frac{\pi}{8} \breve{a}^{*}, \quad \mathrm{e}^{S} \breve{a}^{*} \mathrm{e}^{-S}=\cos \frac{\pi}{8} \breve{a}^{*}+i \sin \frac{\pi}{8} \breve{a} .
$$

Hence, by similar arguments to those in the proof of Theorem 3.1, we get

$$
\mathrm{e}^{S} \sqrt{2}\left(2 \breve{a}^{*} \breve{a}+I\right) \mathrm{e}^{-S}=2 \breve{a}^{*} \breve{a}+i\left(\breve{a}^{* 2}+\breve{a}^{2}\right)+I=M .
$$

We have shown that for $M_{0}=\sqrt{2}\left(2 \breve{a}^{*} \breve{a}+I\right)$, we obtain $\mathrm{e}^{S} M_{0} \mathrm{e}^{-S}=M$. We also have $\mathrm{e}^{-S} M_{0} \mathrm{e}^{S}=M^{*}$, or equivalently,

$$
M_{0}=\mathrm{e}^{-S} M \mathrm{e}^{S}=\mathrm{e}^{S} M^{*} \mathrm{e}^{-S} .
$$


Therefore

$$
\mathrm{e}^{-2 S} M=M^{*} \mathrm{e}^{-2 S}
$$

REMARK 3.6. A left eigenvector of $M$ satisfies

$$
w_{j}^{*} M=\sqrt{2}(1+2 j) w_{j}^{*}, \quad j=0,1,2, \ldots, \quad w_{0}^{*} \breve{c}^{\ddagger}=0, \quad w_{j}^{*} \breve{c}=\kappa_{j} w_{j+1}^{*},
$$

for some $\kappa_{j}>0$, and is such that

$$
\begin{aligned}
& w_{j}=K_{j}\left(\breve{a}^{*}-i \gamma \breve{a}\right)^{j}\left(1,0, i \gamma \sqrt{\frac{1}{2}}, 0,(i \gamma)^{2} \sqrt{\frac{1 \cdot 3}{2 \cdot 4}}, 0,(i \gamma)^{3} \sqrt{\frac{1 \cdot 3 \cdot 5}{2 \cdot 4 \cdot 6}} \cdots\right)^{T} \in l^{2}, \\
& \gamma=\tan \frac{\pi}{8} .
\end{aligned}
$$

Moreover, for an appropriate $K_{j}$, we have $w_{j}^{*} v_{l}=\delta_{k l}$, i.e., the left and right eigenvectors are orthonormal.

We recall that an $n \times n$ matrix $U$ is called $J$-unitary if $U J U^{*} J=J U^{*} J U=I_{n}$, where $I_{n}$ denotes the identity matrix of size $n$ and $J$ is a Hermitian involution with signature $(r, n-r)$. The $J$-unitary matrices form a locally compact group called the J-unitary group.

TheOREm 3.7. Let $J=I_{r} \oplus I_{n-r}$ and let $A$ be an $n \times n$-Hermitian matrix diagonalizable under a J-unitary similarity. Assume that $A$ has distinct eigenvalues. Then there exists a (non-unique) positive definite Hermitian operator $Q$ such that $Q A$ is Hermitian.

Proof. Under the hypothesis, the eigenvalues of $A$ are real. Let $\alpha_{1}, \ldots, \alpha_{n}$ be (the distinct) eigenvalues of $A$. Denote by $x_{1}, \ldots, x_{n}$ and $y_{1}, \ldots, y_{n}$ the associated eigenvectors of $A$ and $A^{*}$, respectively; that is,

$$
A x_{j}=\alpha_{j} x_{j}, \quad A^{*} y_{j}=\alpha_{j} y_{j}, \quad j=1, \ldots, n
$$

By straightforward computation, it follows that

$$
y_{j}^{*} x_{k}=0, \quad \text { for } \quad j \neq k, \quad j, k=1, \ldots, n \text {. }
$$

Assume the eigenvectors $x_{j}$ are normalized according to $x_{j}^{*} J x_{j}=1$ for $j=1, \ldots, r$, and $x_{j}^{*} J x_{j}=-1$ for $j=r+1, \ldots, n$. We chose $y_{j}=J x_{j}$ for $j=1, \ldots, r$, and $y_{j}=-J x_{j}$ for $j=r+1, \ldots, n$. Then, we have $y_{j}^{*} x_{j}=1, j=1, \ldots, n$. Consider the matrix $Q$ defined by $y_{j}=Q x_{j}, j=1, \ldots, n$ This matrix is Hermitian, because $x_{j}^{*} Q x_{k}=\delta_{j k}$ and for an arbitrary vector $z=\sum_{j_{1}}^{n} c_{j} x_{j}$ we find $z^{*} Q^{*} z=z^{*} Q z$. We also get $z^{*} Q z=\sum_{j=1}^{n}\left|c_{j}\right|^{2}>0$, and so $Q$ is positive definite. Moreover, $Q A$ is Hermitian. In fact, 


$$
x_{j}^{*} Q A x_{k}=y_{j}^{*} A x_{k}=\alpha_{j} y_{j}^{*} x_{k}=\alpha_{j} \delta_{j k} .
$$

Considering an arbitrary vector $z=\sum_{j=1}^{n} c_{j} x_{j}$, we find that $z^{*} Q A z=\sum_{j=1}^{n} \bar{c}_{j} c_{j} \alpha_{j}$ is real. So the result clearly follows.

4. Final Remarks. The above theorem still holds under the assumption of the eigenvalues of the matrix not being distinct. This can be easily seen by perturbation and continuity arguments.

We observe that the matrix $M_{\beta}$ in (3.3) is J-Hermitian for $J=I_{1} \oplus\left(-I_{1}\right) \oplus I_{1} \oplus$ $\left(-I_{1}\right) \oplus \cdots$, while the matrix $M_{\theta}$ in $(3.7)$ is $J$-Hermitian for $J=I_{2} \oplus\left(-I_{2}\right) \oplus I_{2} \oplus$ $\left(-I_{2}\right) \oplus \cdots$. The eigenvalues of $J$-Hermitian matrices are real numbers and pairs of complex conjugate numbers (see, e.g., $[1,2]$ ). If we consider finite matrices of the types (3.3) or (3.7), then complex conjugate eigenvalues may appear.

It would be of interest to answer the following questions:

1) Does the last theorem still hold for infinite matrices of the type considered here?

2) What can be said about the eigenvalues and eigenvectors of the matrices in $\mathcal{R}$ when we take $\alpha$ nonreal?

Acknowledgments. The authors thank the Referee for crucial observations.

\section{REFERENCES}

[1] T. Ando, Linear operators on Krein spaces, Lecture Notes, Hokkaido University, Sapporo, Japan, 1979.

[2] T. Ya. Azizov, I. S. Iokhvidov, Linear Operators in Spaces with an Indefinite Metric, Nauka, Moscow, 1986, English Translation: Wiley, New York, 1989.

[3] Bender CM, Making sense of non-Hermitian Hamiltonians, Rep. Prog. Phys., 70:947-1018, 2007.

[4] R. Bhatia, Matrix Analysis, Springer-Verlag, New York, 1997.

[5] R.G. Cooke, Infinite Matrices and Sequences Spaces, Dover, New York, 1955.

[6] Jan Dereziński, Unbounded linear operators, Lecture Notes, in http://www.fuw.edu.pl/ derezins/mat-u.pdf.

[7] A.S. Davidov, Quantum Mechanics, Pergamon Press, Oxford, 1976.

[8] H. F. Jones, On pseudo-Hermitian Hamiltonians and their Hermitian counterparts, J. Phys. A: Math. Gen. 38:1741-1746, 2005.

[9] M. S. Swanson, Transition elements for a non-Hermitian quadratic Hamiltonian, J. Math. Phys. 45:585-601, 2004. 